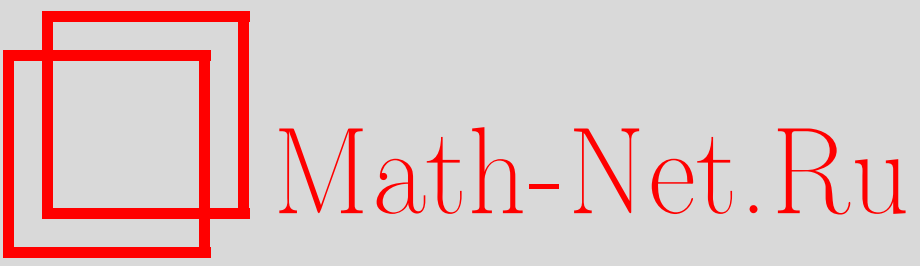

М. И. Тихомирова, В. П. Чистяков, Статистический критерий типа критерия пустых ящиков, Матем. вопр. криптогр., 2010, том 1, выпуск 1, 101-108

DOI: https://doi.org/10.4213/mvk6

Использование Общероссийского математического портала Math-Net.Ru подразумевает, что вы прочитали и согласны с пользовательским соглашением

http://www . mathnet.ru/rus/agreement

Параметры загрузки:

IP : 54.224 .60 .19

26 апреля 2023 г., 17:43:29 
УДК 519.237.32

\title{
Статистический критерий типа критерия пустых ящиков
}

\author{
М. И. Тихомирова ${ }^{1)}$, В. П. Чистяков ${ }^{2)}$ \\ 1) Академия криптографии Российской Федерации, Москва \\ ${ }^{2)}$ Математический институт им. В. А. Стеклова РАН, Москва
}

Получено 22.IV.2010

Рассматриваются два статистических критерия для проверки простых статистических гипотез о последовательности независимых испытаний с конечным числом исходов: критерий со статистикой, равной числу непоявившихся сплошных цепочек одинаковых исходов, и критерий со статистикой, равной числу испытаний до появления заданного числа разных цепочек одинаковых исходов. Критерии могут быть полезными для обнаружения зависимостей между результатами испытаний.

Ключевые слова: критерий пустых ящиков, статистические критерии.

\section{The statistical test of the empty cells type}

\section{I. Tikhomirova ${ }^{1)}$, V. P. Chistyakov ${ }^{2)}$ \\ ${ }^{1)}$ Academy of Cryptography of Russian Federation, Moscow \\ ${ }^{2)}$ Steklov Mathematical Institute of RAS, Moscow}

\begin{abstract}
Two tests for testing simple hypotheses on a sequence of independent trials with finite number of outcomes are considered. The first test is based on the number of non-appeared tuples consisting of repeated outcome. The second test is based on the number of trials until the appearance of a given number of different tuples of such type. These tests may be used to detect dependencies between observations.
\end{abstract}

Key words: empty cells test, simple hypotheses.

Citation: Mathematical Aspects of Cryptography, 2010, vol. 1, no. 1, pp. 101-108 (Russian). 


\section{1. Постановка задачи}

Рассмотрим последовательность

$$
x_{1}, x_{2}, \ldots, x_{t}, \ldots, \quad x_{t} \in \mathfrak{N}=\{1, \ldots, N\},
$$

независимых одинаково распределенных случайных величин. По гипоте3e $H_{0}$

$$
\mathbf{P}\left\{x_{t}=k\right\}=\frac{1}{N}, \quad k=1, \ldots, N ;
$$

по конкурирующей гипотезе $H_{1}$

$$
\mathbf{P}\left\{x_{t}=k\right\}=p_{k}>0, \quad k=1, \ldots, N, \quad \sum_{k=1}^{N} p_{k}=1 .
$$

Обозначим через $\mu_{0}\left(n, B_{0}\right)$ число непоявившихся $s$ - цепочек из множества

$$
B_{0}=\left\{\left(i_{1}, \ldots, i_{s}\right): \quad i_{1}=\ldots=i_{s}=i, \quad i \in \boldsymbol{\aleph}\right\}
$$

в выборке

$$
x_{1}, x_{2}, \ldots, x_{n+s-1},
$$

образованной $n+s-1$ первыми членами последовательности (1). Используем статистику $\mu_{0}\left(n, B_{0}\right)$ для построения критерия, различающего гипотезы $H_{0}$ и $H_{1}$.

При $s=1$ статистика $\mu_{0}\left(n, B_{0}\right)$ является статистикой известного критерия пустых ящиков (см. [1]). Обобщения этого критерия при $s>1$ со статистикой $\mu_{0}(n, B)$, где $B$ является множеством всех $s$-цепочек, рассматривались в работе [2]. Результаты работ [3, 4] позволяют исследовать критерий со статистикой $\mu_{0}\left(n, B_{0}\right)$.

Будем исследовать этот критерий в схеме серий, когда при $n, N \rightarrow \infty$ выполняются следующие условия:

$$
\begin{gathered}
p_{k}=\frac{1}{N}\left(1+\frac{b_{k}(N)}{\sqrt[4]{N}}\right), \quad\left|b_{k}(N)\right| \leq B<\infty, \quad k \in \boldsymbol{\aleph}, \\
b_{N}^{2}=\frac{1}{N} \sum_{k=1}^{N} b_{k}^{2}(N) \rightarrow b^{2},
\end{gathered}
$$




$$
\alpha_{N}=\frac{n}{N^{s}} \rightarrow \alpha, \quad 0<\alpha<\infty, \quad s=\text { const }, \quad s \geq 2 .
$$

Величина $b^{2}=0$ при гипотезе $H_{0}$ и $b^{2}>0$ при гипотезе $H_{1}$.

В условиях (6) исследуется также статистика $v_{k}(N)$, равная числу первых членов последовательности (1), среди которых впервые появилось $k$ различных $s$ - цепочек из множества $B_{0}$.

Зависимость статистик $\mu_{0}(n, N)$ и $v_{k}(N)$ от цепочек длины $s$ позволяет предположить, что соответствующие критерии могут выявлять зависимости, появляющиеся на цепочках длины $s$. Для вычисления $\mu_{0}(n, N)$ и $v_{k}(N)$ требуется значительно меньший объем памяти по сравнению с аналогичными статистиками, определяемыми по полному множеству возможных значений $s$ - цепочек. Это позволяет увеличить $s$ и выявлять зависимости, проявляющиеся на более длинных цепочках.

\section{2. Статистический критерий с $\mu_{0}\left(n, B_{0}\right)$}

Распределения вероятностей при гипотезах $H_{0}$ и $H_{1}$ будем обозначать через $\mathbf{P}_{0}$ и $\mathbf{P}_{1}$ соответственно. Из результатов работы [4] следует, что при $n, N \rightarrow \infty$ в схеме серий (6), (7)

$$
\mathbf{P}_{1}\left\{\frac{\mu_{0}\left(n, B_{0}\right)-N a_{1}(N)}{\sigma_{1}(N) \sqrt{N}}<x\right\} \rightarrow \Phi(x)=\frac{1}{\sqrt{2 \pi}} \int_{-\infty}^{x} e^{-\frac{u^{2}}{2}} d u,
$$

где

$$
a_{1}(N)=\frac{1}{N} \sum_{k=1}^{N} e^{-\alpha_{N}\left(N p_{k}\right)^{s}}, \quad \sigma_{1}^{2}(N)=\sigma^{2}(N)=e^{-\alpha_{N}}\left(1-e^{-\alpha_{N}}\right) .
$$

Отсюда следует, что при гипотезе $H_{0}$ статистика $\mu_{0}\left(n, B_{0}\right)$ асимптотически нормальна с параметрами $\left(N a_{0}(N), N \sigma_{0}^{2}(N)\right)$, где

$$
a_{0}(N)=e^{-\alpha_{N}}, \quad \sigma_{0}^{2}(N)=\sigma^{2}(N) .
$$

Будем принимать гипотезу $H_{1}$, если

$$
\mu_{0}\left(n, B_{0}\right)>C,
$$

где

$$
C=N e^{-\alpha_{N}}+u_{\varepsilon_{0}} \sigma \sqrt{N}, \quad \sigma^{2}=e^{-\alpha}\left(1-e^{-\alpha}\right),
$$




$$
1-\Phi\left(u_{\varepsilon}\right)=\varepsilon
$$

в противном случае принимается гипотеза $H_{0}$.

Вероятности $\varepsilon_{0}(N), \varepsilon_{1}(N)$ ошибок первого и второго родов критерия (11) определяются равенствами

$$
\varepsilon_{0}(N)=\mathbf{P}_{0}\left\{\mu_{0}\left(n, B_{0}\right)>C\right\}, \quad \varepsilon_{1}(N)=\mathbf{P}_{1}\left\{\mu_{0}\left(n, B_{0}\right) \leq C\right\} .
$$

Найдем предельные значения вероятностей (14) при $n, N \rightarrow \infty$ в схеме серий (6), (7). Докажем сначала следующее утверждение.

Лемма 1. При $n, N \rightarrow \infty$ в схеме серий (6), (7)

$$
a_{1}(N)=e^{-\alpha_{N}}\left(1+\frac{s \alpha_{N}\left(1+\alpha_{N} s-s\right)}{2 \sqrt{N}}-b_{N}^{2}\right)+O\left(\frac{1}{N^{3 / 4}}\right) .
$$

Доказательство. Используя (6), представим $a_{1}(N)$ в следующем виде:

$$
a_{1}(N)=\frac{1}{N} e^{-\alpha_{N}} \sum_{k=1}^{N} e^{-\alpha_{N}}\left(\left(1+\frac{b_{k}(N)}{\sqrt[4]{N}}\right)^{s}-1\right) .
$$

Отсюда и из равенств

$$
\begin{gathered}
\left(1+\frac{b_{k}(N)}{\sqrt[4]{N}}\right)^{s}-1=\frac{s b_{k}(N)}{\sqrt[4]{N}}+\frac{s(s-1) b_{k}^{2}(N)}{2 \sqrt{N}}+O\left(\frac{1}{N^{3 / 4}}\right) \\
\sum_{k=1}^{N} b_{k}(N)=0
\end{gathered}
$$

следует утверждение леммы.

Теорема 1. При $n, N \rightarrow \infty$ в схеме серий (6), (7) существуют предель

$$
\lim _{N \rightarrow \infty} \varepsilon_{0}(N)=\varepsilon_{0}, \quad \lim _{N \rightarrow \infty} \varepsilon_{1}(N)=\varepsilon_{1},
$$

где $\varepsilon_{1}$ определяется из равенства

$$
u_{\varepsilon_{0}}+u_{\varepsilon_{1}}=\frac{\alpha s(1+s \alpha-s)}{2 \sigma} e^{-\alpha} b^{2},
$$

$u_{\varepsilon_{0}}, u_{\varepsilon_{1}}$ определены равенством (13), $\sigma^{2}=e^{-\alpha}\left(1-e^{-\alpha}\right)$.

Доказательство. Из первого равенства (14) и равенства (12) следует, что

$$
\varepsilon_{0}(N)=\mathbf{P}_{0}\left\{\frac{\mu_{0}\left(n, B_{0}\right)-N a_{0}(N)}{\sigma \sqrt{N}}<u_{\varepsilon_{0}}\right\}
$$


Отсюда и из асимптотической нормальности $\mu_{0}\left(n, B_{0}\right)$ с параметрами (10) при гипотезе $H_{0}$ получаем, что $\lim _{N \rightarrow \infty} \varepsilon_{0}(N)=\varepsilon_{0}$.

Из второго равенства (14) получаем:

$$
\varepsilon_{0}(N)=\mathbf{P}_{1}\left\{\frac{\mu_{0}\left(n, B_{0}\right)-N a_{1}(N)}{\sigma \sqrt{N}}>\frac{C-N a_{1}(N)}{\sigma \sqrt{N}}\right\} .
$$

Используя лемму 1 и равенство (12), находим:

$$
\begin{aligned}
& \frac{C-N a_{1}(N)}{\sigma \sqrt{N}}=\frac{N\left(a_{0}(N)-a_{1}(N)\right)+u_{\varepsilon_{0}} \sigma \sqrt{N}}{\sigma \sqrt{N}}= \\
& =u_{\varepsilon_{0}}-e^{-\alpha_{N}} \frac{s \alpha_{N}\left(1+s \alpha_{N}-s\right)}{2 \sigma} b_{N}^{2}+O\left(\frac{1}{\sqrt[4]{N}}\right) .
\end{aligned}
$$

Отсюда следует, что $\lim _{N \rightarrow \infty} \varepsilon_{1}(N)=\varepsilon_{1}$, где

$$
-u_{\varepsilon_{1}}=u_{\varepsilon_{0}}-e^{-\alpha} \frac{b^{2}}{2} \frac{s \alpha(1+s(\alpha-1))}{\sigma} .
$$

Теорема доказана.

\section{3. Предельное распределение статистики $v_{k}(N)$}

Асимптотическая нормальность $v_{k}(N)$ при гипотезе $H_{0}$ установлена в работе [3]. В доказательстве использовалось представление $v_{k}(N)$ в виде суммы независимых слагаемых. В неравновероятном случае такого представления нет. Для доказательства асимптотической нормальности $v_{k}(N)$ в неравновероятном случае воспользуемся установленной в работе [4] асимптотической нормальностью $\mu_{0}\left(n, B_{0}\right)$ и равенством

$$
\mathbf{P}\left\{v_{k}(N) \leq n\right\}=\mathbf{P}\left\{\mu_{0}\left(n, B_{0}\right) \leq N-k\right\} .
$$

Будем предполагать, что при $N, k \rightarrow \infty$ выполняется условие

$$
\gamma_{N}=\frac{k}{N} \rightarrow \gamma, \quad 0<\gamma<1
$$

Выберем $n$ так, чтобы при условии (16)

$$
N-k=N a_{1}(N)+x_{N} \sigma(N) \sqrt{N}, \quad x_{N} \rightarrow x .
$$


Лемма 2. Если при $N, k \rightarrow \infty$ выполнены условия (16), (6) и пизменяется так, что выполняется равенство (17), то

$$
\alpha_{N}=\frac{n}{N^{s}} \rightarrow \alpha=-\ln (1-\gamma), \quad 0<\alpha<\infty .
$$

Доказательство. Отметим, что

$$
e^{-\alpha_{N}} \leq 1, \quad \sigma^{2}(N) \leq 1, \quad e^{-\alpha_{N}\left(N p_{k}\right)^{s}} \leq 1 .
$$

Из равенства (17) следует равенство

$$
a_{1}(N)=1-\gamma_{N}-x_{N} \frac{\sigma(N)}{\sqrt{N}} .
$$

Отсюда, используя (18), получим:

$$
\frac{1}{N} \sum_{k=1}^{N} e^{-\alpha_{N}\left(1+\frac{b_{k}(N)}{\sqrt[4]{N}}\right)^{s}}=1-\gamma_{N}+O\left(\frac{1}{\sqrt{N}}\right) .
$$

Если выполнены условия (16), то при $n, N \rightarrow \infty$ правая часть равенства (19) стремится к $1-\gamma$. Таким образом, существует

$$
\lim _{N \rightarrow \infty} \frac{1}{N} \sum_{k=1}^{N} e^{-\alpha_{N}\left(1+\frac{b_{k}(N)}{\sqrt[4]{N}}\right)^{s}}=1-\gamma .
$$

Отсюда следует утверждение леммы.

Теорема 2. Если выполнены условия (6) $u$ (16), то при $N, k \rightarrow \infty$

$$
\mathbf{P}\left\{\frac{v_{k}(N)-A(N)}{D(N)}<x\right\} \rightarrow \Phi(x),
$$

где

$$
\begin{gathered}
A(N)=N^{s} \ln \frac{1}{1-\gamma_{N}}-\sqrt{N^{2 s-1}} \frac{b^{2}}{2}\left(1+(s-1) \ln \frac{1}{1-\gamma_{N}}\right) \ln \frac{1}{1-\gamma_{N}}, \\
D^{2}(N)=N^{2 s-1} \frac{\gamma_{N}}{1-\gamma_{N}} .
\end{gathered}
$$

Доказательство. Определим $n$ равенством (17). Из леммы 2 следует, что выполнены условия асимптотической нормальности $\mu_{0}\left(n, B_{0}\right)$ и, следовательно, правая часть равенства (15) стремится к $\Phi(x)$. Найдем теперь асимптотическую формулу для $n$. 
Положим $z_{N}=e^{-\alpha_{N}}$. Используя лемму 1 и равенство (17), получим:

$$
\begin{gathered}
z_{N}=1-\gamma_{N}-\frac{1}{\sqrt{N}}\left(-z_{N} \frac{b_{N}^{2} s\left(1-(s-1) \ln z_{N}\right) \ln z_{N}}{2}+x_{N} \sqrt{z_{N}\left(1-z_{N}\right)}\right)+g_{N}\left(z_{N}\right), \\
g_{N}\left(z_{N}\right)=O\left(\frac{1}{N^{3 / 4}}\right)
\end{gathered}
$$

где

$$
g_{N}\left(z_{N}\right)=a_{1}(N)-z_{N}\left(1+\frac{s \alpha_{N}\left(s \alpha_{N}-s+1\right)}{2 \sqrt{N}} b_{N}^{2}\right) .
$$

Положим

$$
F(z)=1-\gamma_{N}+\frac{1}{\sqrt{N}}\left(z \frac{1}{2} b_{N}^{2} s\left(1-(s-1) \ln z_{N}\right) \ln z_{N}-x_{N} \sqrt{z(1-z)}\right)+g_{N}\left(z_{N}\right) .
$$

Равенство (21) можно записать в виде $z_{N}=F\left(z_{N}\right)$ и, следовательно,

$$
\begin{gathered}
z_{N}=F\left(F\left(z_{N}\right)\right)= \\
=\left(1-\gamma_{N}\right)\left(1-\frac{1}{\sqrt{N}}\left(\sqrt{\frac{\gamma_{N}}{1-\gamma_{N}}}+\frac{b_{N}^{2}}{2}\left(1+(s-1) \ln \frac{1}{1-\gamma_{N}}\right) s \ln \frac{1}{1-\gamma_{N}}\right)\right)+O\left(\frac{1}{N^{3 / 4}}\right) .
\end{gathered}
$$

Отсюда

$$
\begin{gathered}
\alpha_{N}=\frac{n}{N^{s}}=\ln \frac{1}{1-\gamma_{N}}+ \\
+\frac{1}{\sqrt{N}}\left(\frac{b_{N}^{2}}{2}\left(1+(s-1) \ln \frac{1}{1-\gamma_{N}}\right)+\sqrt{\frac{\gamma_{N}}{1-\gamma_{N}}}\right)+O\left(\frac{1}{N^{3 / 4}}\right) .
\end{gathered}
$$

Используя это равенство, получим указанную нормировку и центрировку $v_{k}(N)$ в левой части равенства (15). Теорема доказана.

Критерий со статистикой $v_{k}(N)$ определим аналогично критерию (11): если

$$
v_{k}(N)<C,
$$

где

$$
C=N^{s} \ln \frac{1}{1-\gamma_{N}}-u_{\varepsilon_{0}} \sqrt{N^{2 s-1} \frac{\gamma_{N}}{1-\gamma_{N}}},
$$

то принимается гипотеза $H_{1}$; в противном случае принимается гипотеза $H_{0}$. 
Если выполнены условия (6) и (16), то вероятности ошибок $\varepsilon_{0}(N)=$ $=\mathbf{P}_{H_{0}}\left(v_{k}<C\right), \varepsilon_{1}(N)=\mathbf{P}_{H_{1}}\left(v_{k}>C\right)$ критерия (22) при $N, k \rightarrow \infty$ сходятся:

$$
\lim _{N \rightarrow \infty} \mathcal{E}_{v}(N)=\varepsilon_{v}, \quad v=0,1,
$$

И

$$
u_{\varepsilon_{0}}+u_{\varepsilon_{1}}=\frac{1}{2} b^{2} \frac{\left(1+(s-1) \ln \frac{1}{1-\gamma}\right) \ln \frac{1}{1-\gamma}}{\sqrt{\frac{\gamma}{1-\gamma}}} .
$$

\section{Список литературы}

1. Колчин В. Ф., Севастьянов Б. А., Чистяков В. П. Случайные размещения. - М.: Наука, 1964.

2. Тихомирова М. И., Чистяков В. П. О статистических критериях отсутствующих $s$-грамм. - В сб.: Труды по дискретной математике. Т. 7. - М.: ФИЗМАТЛИТ, 1997, с. 265-278.

3. Тихомирова М. И. Предельные распределения числа непоявившихся цепочек одинаковых исходов. - Дискрет. матем., 2008, т. 20, в. 3, с. 40-46.

4. Тихомирова М. И. Асимптотическая нормальность числа непоявившихся несплошных цепочек исходов независимых испытаний. - Дискрет. матем., 2009, т. 21, в. 2, с. 112-125. 